\title{
Impacts of Meso-structure and Organic Loadings of Fluoroalcohol Derivatives/SBA-15 Hybrids on Nerve Agent Simulant Sensing
}

\author{
Qi Zheng, Huimin Li, Zhixuan Cheng, Jiaqiang. Xu \\ Department of Chemistry, College of Science, Shanghai University, 99 Shangda Road, Shanghai \\ 200444, China, \\ xujiaqiang@shu.edu.cn
}

\begin{abstract}
:
This paper uses the measurement of quartz crystal microbalance (QCM) to investigate the effects of fluoroalcohol derivatives/SBA-15 hybrids with varied mesopore structures and organic loadings on the sensing behavior of nerve agent simulant DMMP. The results show that much faster and higher response value is achieved when the hybrid materials have relative higher surface area and pore volume. It is especially true when detecting DMMP in a ppb level. On the other hand, mesoporous hybrids with lower loadings of fluoroacohol derivatives show weaker selective properties. The specific interaction with DMMP vapor is greatly enhanced as the amount of fluoroalcohol derivatives increases. Therefore, in the fluoroalcohol derivatives functionalized SBA-15 materials, pore ordering and organic loadings are clearly important factors determining QCM-based DMMP sensing behavior.
\end{abstract}

Key words: fluoroalcohol derivatives/SBA-15 hybrids, nerve agent sensing, quartz crystal microbalance (QCM), impacts of meso-structure and organic loadings

\section{Introduction}

Establishing lower-power, high-performance chemical vapor detection for defense, homeland security is currently one of the most urgent and important issues, especially for detection of warfare agents, such as Sarin, known to be a fatal nerve agent. Novel materials, including carbon nanotubes[1], graphene[2], metal oxides[3], have gained considerable attention as effective active materials for chemical sensing due to their outstanding electrical, thermal, and mechanical properties. On the other hand, porous materials such as mesoporous silicas with high specific surface areas and regular pore geometries providing an amplified target-receptor interface, enabling them desirable for chemical sensing.[4] Therefore, the interface between the material and analytes is crucial to sensing performance. Ongoing efforts have been made to illustrate the contribution of several factors, including surface functionality, pore structure, and functional group distribution, to the selectivity and sensitivity of a sensor response.

Recently, we have developed a highperformance nerve agent simulant dimethyl methylphosphonate (DMMP) sensor with organic-inorganic mesoporous SBA-15 hybrids based on quartz crystal microbalance (QCM) devices.[5] The guest selectivity of assynthesized materials has been tuned by incorporating hexafluoroisopropanol (HFIP) derivatives into silica frameworks, resulting designable selective sensing toward DMMP vapor. In this work, efforts have been focused on investigating the influence of mesopore structure and functionality loadings on QCMbased DMMP sensing behavior. The results show that mesopore geometry of the mesoporous hybrid is major factor in sensing efficiency; while incorporation of different percentages of functionality has been shown to lead to different selectivity toward DMMP molecules.

\section{Experimental Section}

All chemicals were used without additional purification. Poly(ethylene oxide)poly(propylene oxide)-poly(ethylene oxide) $\left(\left(\mathrm{P} 123, \quad \mathrm{EO}_{20}-\mathrm{PO}_{70}-\mathrm{EO}_{20}, \quad \mathrm{M}_{\mathrm{w}}=5800\right)\right.$ was purchased from Sigma Aldrich. Carboxyethylsilanetriol sodium salt (CES, 25\% in water) was obtained from Gelest. Hexafluoroacetone trihydrate was obtained from Aladdin. All the other reagents (analyticalgrade purity) were purchased from Shanghai Chemical Reagent Co. Ltd. (Shanghai, China). 
2.1 Synthesis of fluoroalcohol derivative/SBA15 hybrids containing hexafluoroisopropanol (HFIP) substituents

The mesoporous SBA-15 hybrid covalently functionalized by the HFIP was achieved by in situ amidation reaction between carboxylic acid groups functionalized SBA-15 and phexafluoroisopropanol aniline (HFIPA). (see Scheme 1)

Firstly, $-\mathrm{COOH}$ functionalized SBA-15 was synthesized in a similar way as described in ref. [6]. The composition of the reaction mixture was $\mathrm{x} / 100$ CES : $(100-\mathrm{x}) / 100$ TEOS : 0.0168 P123 : $5.85 \mathrm{HCl}: 162.68 \mathrm{H}_{2} \mathrm{O}$. The $\mathrm{x}$ is $3,10,20$.

Secondly, corresponding amount of HFIPA [7] was used to react with the preceding attached carboxylic acid groups and thus led to the HFIP groups being bonded on SBA-15 pore surface. The as-made samples with initial amount of $\mathrm{COOH}$ ranges from 3 to 10 were denoted as $\mathrm{S} 1$, S2 and S3, respectively.

\subsection{Methods of characterization}

Crystal structure of the products was identified by powder X-ray diffraction (XRD) analysis using a Dmax 2550V diffractometer with $\mathrm{Cu} \mathrm{K} \propto$ radiation ( $\lambda=1.54056 \AA)$ (Rigaku, Tokyo, Japan), and the XRD data were collected at a scanning rate of $0.02^{\circ} \mathrm{s}^{-1}$ for $2 \theta$ in a range of 0 $10^{\circ}$. Pore structures of the samples were characterized by a transmission electron microscopy (TEM, JEOL JEM-200CX working at $160 \mathrm{kV}$ ). The nitrogen sorption isotherms were measured at $-196{ }^{\circ} \mathrm{C}$ by using a Micromeritics ASAP 2020 system. Surface functionalization was monitored by Fourier transformation infrared spectroscopy (FTIR) with a AVATRA370 FTIR instrument using $\mathrm{KBr}$ plates within $4500-400 \mathrm{~cm}^{-1}$.

\subsection{Sensor configuration and experiment setup}

The chemical sensing experiments were performed by using a lab-made setup that we reported.[5] In order that they could act as electrodes, the QCM resonators (Chenjing Electronic Co., China) used were coated silver electrodes by vapour deposition. The resonance frequency was $10^{7} \mathrm{~Hz}$ (AT-cut) and frequency decreased $(-\Delta F)$ proportionately with an increase in mass $(\Delta \mathrm{m})$ according to the Sauerbrey equation.[8]

\section{Results and discussions}

\subsection{Materials characterization}

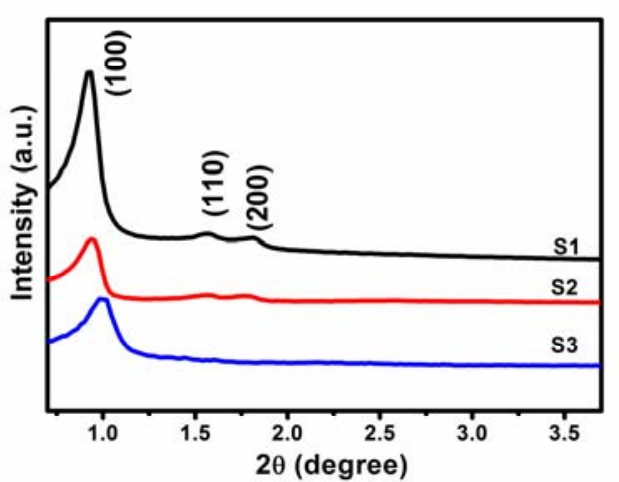

Fig. 1. Small angle XRD patterns for as-made HFIP functionalized mesoporous SBA-15.XRD

pattern of the hexagonal lamelliform mesoporous SBA-15 (Fig. 1) shows three diffraction peaks that can be indexed to (100), (110) and (200) reflections. The observation is in agreement with that of previous reports [9], indicating the hexagonal lamelliform mesoporous SBA-15 has a two-dimensional (2D) mesostructure $(p 6 \mathrm{~mm})$. The nitrogen sorption isotherms for all the samples are of type IV with clear H1-type hysteresis loops at high relative pressure, which is typical for mesoporous solids with uniform size distributions.[6] Fig. 3 displayed a TEM picture of sample S2 with CES contents of 10 , showing well ordered 2D hexagonal mesostructures. The textural properties of all the samples are summarized in Table 1.

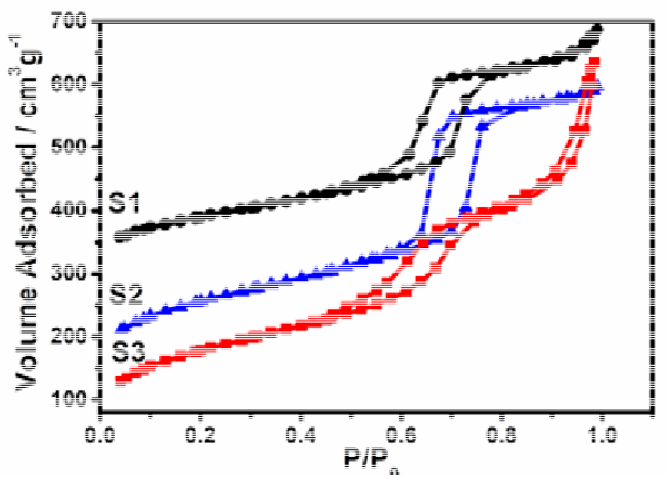

Fig. 2. $\mathrm{N}_{2}$ adsorption isotherms of the as-made HFIP/SBA-15.

The presence of fluoroalcohol derivatives associated with the SBA-15 material was studied by FT-IR spectroscopy (Fig. 4). The formation of a dense silica network is evidenced by the broad bands centered at $1080 \mathrm{~cm}^{-1}$ owing to the bridged oxygen silica bonds between two silicon sites. Besides, the bands located at $800 \mathrm{~cm}^{-1}$ ( $v \mathrm{~s}, \mathrm{Si}-\mathrm{O}-\mathrm{Si}$ ), and $460 \mathrm{~cm}^{-}$ 1 ( $\delta, \mathrm{Si}-\mathrm{O}-\mathrm{Si}$ ), further confirm the existence of the SBA-15 framework. In addition, the stretching vibration bands of the benzene framework at $1519 \mathrm{~cm}^{-1}$, the $-\mathrm{CF}_{3}$ in-plane 
bending vibration band centered at $1380 \mathrm{~cm}^{-1}$, along with the stretching vibration bands of $1650 \mathrm{~cm}^{-1}$ and $1541 \mathrm{~cm}^{-1}$ attributed to $-\mathrm{CONH}-$ groups, indicating that HFIP has been successfully grafted onto the wall of SBA- 15 .

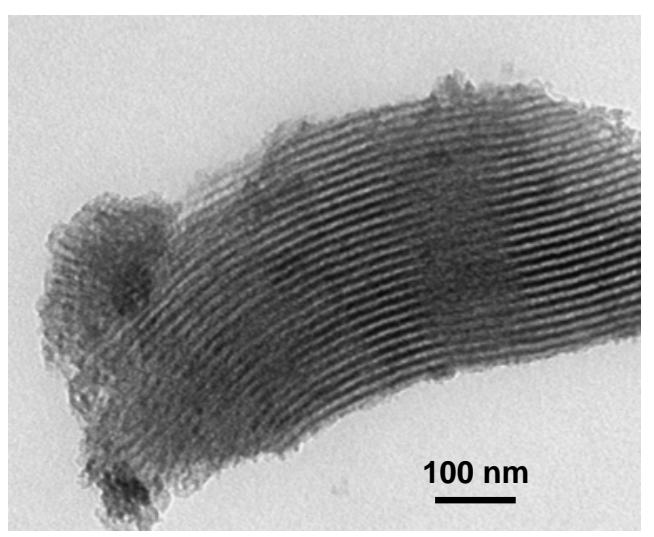

Fig.3. TEM images of HFIP/SBA-15 (S2).

Tab. 1: Textural properties of the as-made HFIP/SBA-15 samples

\begin{tabular}{|c|c|c|c|c|}
\hline sample & $\begin{array}{c}\mathrm{a}_{0} / \\
\mathrm{nm}\end{array}$ & $\begin{array}{c}\mathrm{S}_{\mathrm{BET}} / \\
\mathrm{m}^{2} \mathrm{~g}^{-1}\end{array}$ & $\begin{array}{c}\mathrm{V} / \\
\mathrm{cm}^{3} \mathrm{~g}^{-1}\end{array}$ & $\begin{array}{c}\mathrm{D}_{\mathrm{p}} / \\
\mathrm{nm}\end{array}$ \\
\hline $\mathrm{S} 1$ & 10.88 & 377 & 0.63 & 6.8 \\
\hline $\mathrm{S} 2$ & 9.4 & 681 & 0.85 & 5.0 \\
\hline $\mathrm{S} 3$ & 9.6 & 640 & 0.97 & 5.8 \\
\hline
\end{tabular}

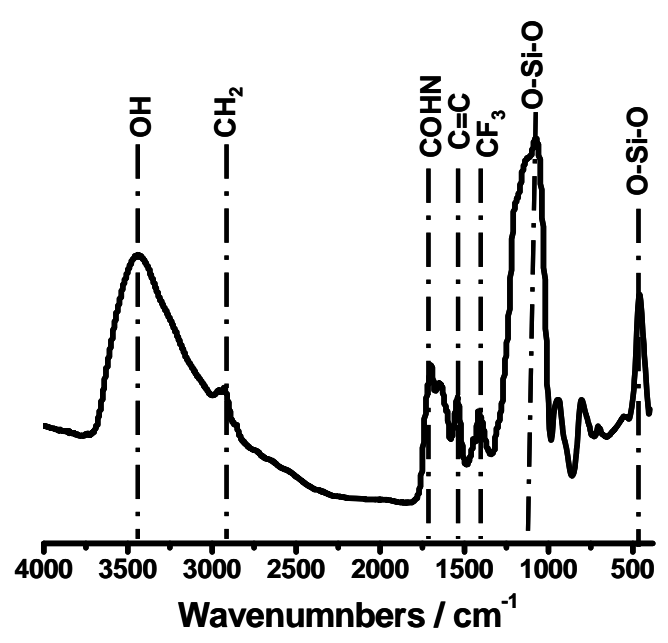

Fig. 4. FT-IR spectra of sample S2.

\subsection{Sensing behaviors of hexafluoroisopropanol (HFIP) derivatives functionalized SBA-15 coated QCM toward DMMP}

After the chemical nature of the powdered samples was evaluated, their responses to DMMP vapour, and the influence of pore structure and functionality distribution were investigated.
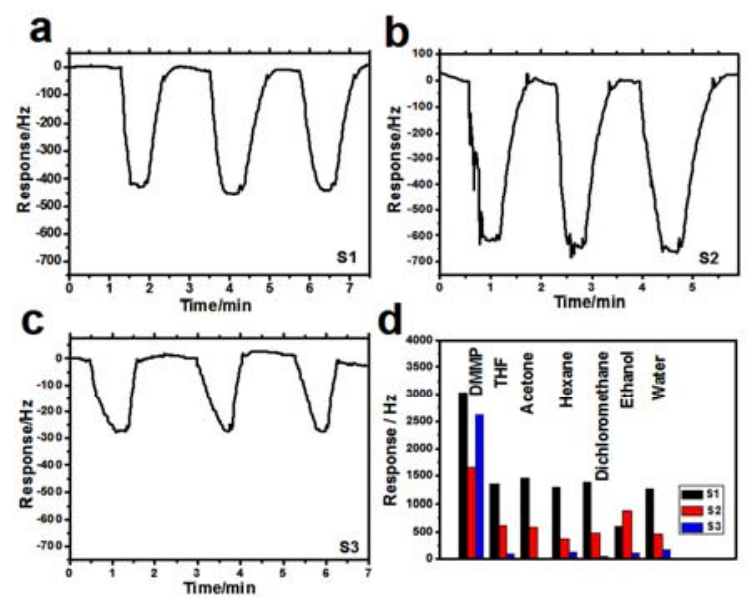

d

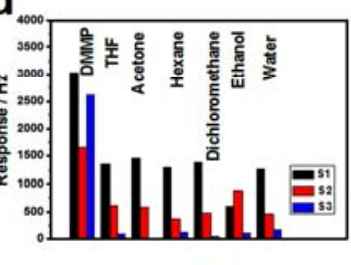

Fig.5. a-c) Short-term repeatable and reversible rapid sensing response to $60 \mathrm{ppm} D M M P$ vapor based on different HFIP/SBA-15 samples (S1, S2, S3). (d) Frequency change of different HFIP/SBA-15 samples coated sensors in response to saturated common organic solvents and 130 ppm DMMP vapor at room temperature.

As shown in Fig. 5a-c, the response curves of HFIP/SBA-15 coated sensors under a concentration of DMMP at $60 \mathrm{ppm}$. All the sensors display fast and reversible responses, however, the S2 based sensor displays around $600 \mathrm{~Hz}$ frequency changes, which is the highest response as compared with the S1 and S3. Fig. 6 gives the sensing behavior of hybrids $\mathrm{S} 1$ and S2 coated sensors toward low concentration of DMMP ranges from $10 \mathrm{ppb}$ to $1 \mathrm{ppm}$. It is clear that S2 has a much shorter response time and enhanced frequency change in unit ppb. From table 1, it can be seen that $S 2$ has the largest surface area and relative high pore volume, which is beneficial to facilitate guest molecules adsorption-desorption process. Therefore, it can be concluded that the mesopore system, ie. surface area and pore volume, plays an important role in enhancing the sensing responses and sensitivity, which is especially true when detecting DMMP in ppb level.
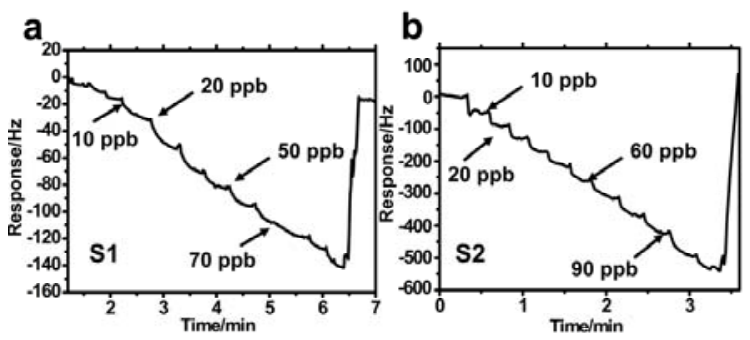

Fig.6. Responses (a)S1 and (b) S2 coated QCM sensors vs DMMP vapor at concentrations ranges from $10 \mathrm{ppb}$ to $1 \mathrm{ppm}$.

The sensor response is another thing to be considered for detection of DMMP. As shown in Fig. 5d, the S3 modified sensor exhibits excellent selectivity property. Therefore, it can 
be demonstrated that the sensor's selectivity behavior depends on loading of organic groups, as S3 has the highest HFIP groups compared with the other two samples.

\section{Conclusion}

To conclude, we have prepared three fluoroalcohol derivatives/SBA-15 hybrids with varied mesopore structures and organic loadings. Their sensing behavior of nerve agent simulant DMMP on the basis of QCM devices has been discussed. It is demonstrated that the mesopore systems including surface area and pore volume of hybrids are major factors in affecting sensing responses and efficiency; on the other hand, incorporation of higher percentages of functionality shown to lead much enhanced selectivity toward DMMP molecules.

\section{Acknowledgements}

The research is supported by the National Nature Science Foundation of China (No. 61071040), and the Leading Academic Discipline Project of Shanghai Municipal Education Commission (J50102).

\section{References}

[1] F. Wang, H. Gu, and T. M. Swager, Carbon Nanotube/Polythiophene Chemiresistive Sensors for Chemical Warfare Agents, J. Am. Chem. Soc 130, 5392-5393 (2008); doi: 10.1021/ja710795k

[2] J. T. Robinson, F. K. Perkins, E. S. Snow, Z Wei, P. E. Sheehan, Reduced Graphene Oxide Molecular Sensors, Nano Letters 8, 3137-3140 (2008); doi:10.1021/nl8013007

[3] S. C. Lee, H. Y. Choi, S. J. Lee, W. S. Lee, J. S. Huh, D. D. Lee, C. Kim, The development of $\mathrm{SnO}_{2}$-based recoverable gas sensors for the detection of DMMP, Sens. Actuators B 137 239245 (2009); doi: 10.1016/j.snb.2008.12.051

[4] B. J. Melde, B. J. Johnson, Mesoporous materials in sensing: morphology and functionality at the meso-interface, Anal. Bioanal. Chem. 398, 15651573 (2010); doi: 10.1007/s00216-010-3688-6

[5] Q Zheng, Y Zhu, J Xu, Z Cheng, H Li, X Li, Fluoroalcohol and fluorinated-phenol derivatives functionalized mesoporous SBA-15 hybrids: high-performance gas sensing toward nerve agent, J. Mater. Chem. 22, 2263-2270 (2012); doi: 10.1039/c1jm14779j

[6] C.-T. Tsai, Y.-C. Pan, C.-C. Ting, S. Vetrivel, A. S. T. Chiang, G. T. K. Fey, H.-M. Kao, A simple onepot route to mesoporous silicas SBA-15 functionalized with exceptionally high loadings of pendnt carboxylic acid groups, Chem. Commun. 33 ,5018-5020 (2009); doi: 10.1039/b09680a

[7] L. Kong, J. Wang, X. Fu, Y. Zhong, F. Meng, T. Luo, J Liu, p-Hexafluoroisopropanol phenyl covalently functionalized single-walled carbon nanotubes for detection of nerve agents, Carbon 48, 1262-1270 (2010); doi:

10.1016/j.carbon.2009.11.051

[8] G. Sauerbrey, The use of quartz oscillators for weighing thin layers and for microweighing, $Z$. Phys. 155, 206-222 (1959)

[9] D.Y. Zhao, Q.S. Huo, J.L. Feng, B.F. Chmelka, G.D. Stucky, Nonionic triblock andstar diblock copolymer and oligomeric surfactant syntheses of highly ordered, hydrothermally stable mesoporous silica structures, J. Am. Chem. Soc. 120, 6024-6036 (1998); doi: 10.1021/ja974025i 